\title{
26. Die günstigste Ionenzusammensetzung des äusseren Mediums für Hydra, mit besonderer Berücksichtigung der antagonistischen Wirkung von $\mathrm{Ca}^{\cdot} \cdot$ gegenüber $K^{\cdot 1)}$.
}

\author{
Von Masutaro Kuwabara. \\ Zoologisches Institut, Fakultät für Naturwissenschaften, \\ Hokkaido Kaiserliche Universität zu Sapporo.
}

(Comm. by N. YATSU, M.I.A., Feb. 12, 1944.)

\section{Einleitung.}

In der Absicht die Beeinflussung der Ionen im äusseren Medium auf die Geschlechtlichkeit der Süsswasserhydroiden zu untersuchen, war es zunächst nötig, die für die Hydren am besten geeignete Zusammensetzung der Ionen des Kulturmediums festzustellen, da bei den Meerwassertieren das künstliche Meerwasser als das Kontrollmedium zum Experiment gebraucht werden kann, bei den Süsswassertieren dagegen ein solches Medium fehlt.

Es ist schon von vielen Autoren nachgewiesen worden, dass bei vielen Arten von Süsswassertieren die günstigsten Lebensbedingungen dann vorliegen, wenn die am meisten interessierenden vier Kationen $\left(\mathrm{Na}{ }^{*}, \mathrm{~K}^{*}, \mathrm{Ca}^{*}, \mathrm{Mg}^{*}\right)$ zugegen sind und bei geeigneter Gesammtkonzentration in dem. Verhältnis zueinander stehen, das dem des Seewassers entspricht. Dabei wird angenommen, dass unter den genannten vier Ionen die einwertigen gegenüber zweiwertigen antagonistisch zueinander wirkend sind, und bei dem dem Seewasser entsprechenden Verhältnis ihre Wirkungen in Gleichgewicht stehen. E. Berger (1929) hat aus seinen Untersuchungen mit Daphnien, Batrachienlarven und Tinca (Schleie) geschlossen, dass bei binären Salzgemischen das optimale Verhältnis von zueinander antagonistisch wirkenden Ionen, also von einwertigen und zweiwertigen, ganz artspezifisch ist, aber bei ternärem und quaternärem Gemisch die Artspezifität verschwindet, dabei stetig das dem Seewasser entsprechende Verhältnis als am besten geeignetes vorliegt.

Tatsächlich aber ist das Verhältnis der Ionenzusammensetzung des Süsswassers, in welchem Hydren gedeihen, von dem des Seewassers weit abweichend. Und nun konnte ich das Medium herstellen, das in Ionenzusammensetzung meinem bisherigen Kulturmedium, Wasserleitungswasser aus der Universität, möglichst ähnlich, ausserdem der Hydra als das dem Seewasser entsprechende Medium günstiger ist. Mir scheint es also, dass das Medium der Zusammensetzung, die der des Seewassers entspricht, auch in der passenden Konzentration nicht immer für alle Arten von Süsswassertieren geeignet ist.

Im Laufe des Versuches konnte ich vor allem die merkwürdige

1) Contribution No. 184 from the Zoological Institute, Faculty of Science, Hokkaido Imperial University, Sapporo, Japan. 
antagonistische Wirkung von $\mathrm{Ca}$ “ gegenüber $\mathrm{K}$ ' auf Hydra feststellen.

An dieser Stelle möchte ich Herrn Prof. Dr. Tohru Uchida den herzlichsten Dank aussprechen, da unter seiner Leitung diese Arbeit gemacht worden ist. Diese Forschung ist zum Teil mit den Stipendien, die von der " Japanischen Gesellschaft zur Förderung der wissenschaftlichen Forschung" dem Verfasser günstigst zur Verfügung gestellt wurden, gemacht.

\section{Methodisches.}

Als Versuchsmaterial wurde Hydra attenuata angewandt. Die Hydren wurden bei den Versuche je 5 zusammen, in Glasflaschen von $5 \mathrm{~cm}$ Durchmesser und $10 \mathrm{~cm}$ Höhe, mit Glas gestöpselt, in $50 \mathrm{cc}$ verschiedener Lösungen ohne Fütterung eine Woche lang gezüchtet. Angreifungsgrade der verschiedenen Lösungen wurden mit dem Grad der Depression der Hydren bestimmt, da die, welche unter ungünstigen Bedingungen sterben, immer die Prozesse der Depression durchgemacht haben. Ich habe zu diesem Zwecke die Prozesse der Depression in folgende 5 Stufen eingeteilt (Abb. 1).

I) Ausbildung des Kügelchens an den Tentakelenden; II) Verkürzung (Zerfall) der Tentakeln bis über halbe Länge; III) Tentakeln als Stummeln um den Mundkegel ausgebildet; IV) Anfang des Zerfalles an der Umgebung des Mundkegels;
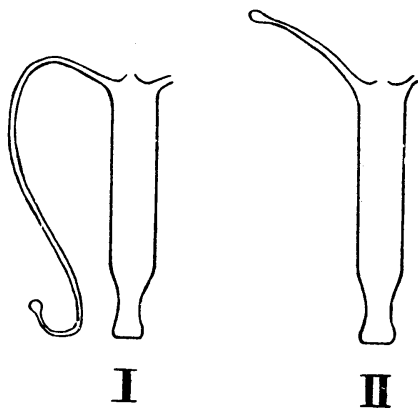

V) Zerfall des ganzen Körpers.

Die Beobachtung wurde täglich zu bestimmter Zeit gemacht, und am siebten Tag des Experimentes wurde die Beeinflussung der Lösungen durch den Grad der Depression bestimmt; bei Verlängerung der Züchtdauer wurde die Hungererscheinung merkbar, und bei Verkürzung derselben erschien die Beeinflussung der Lösung nicht klar. Die Lösungen wurden jeden zweiten Tag mit frischen gewechselt. Für jeder Prüfung einer Lösung wurde je 10 Exemplare, die ständig je 5

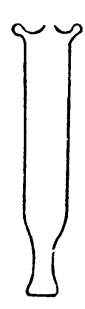

III

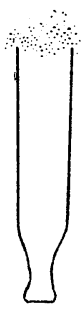

$\mathbb{\nabla}$

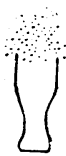

Abb. 1. Die fünf Stufen des Depressionsprozesses. zusammen in zwei gleichen Flaschen gezüchtet wurden, angewandt. Dadurch konnte ich kontrollieren, ob unter vermutlich gleichen Bedingungen die Hydren sich immer gleich verhalten, ausserdem war die Züchtung von 5 Exemplaren äusserst günstig für Beobachtungen.

Die Versuchsmaterialien wurden stets aus einer Klone genommen, mit besonderer Berücksichtigung, möglichst in gleichen Zuständen stehende Exemplare auszuwählen. Etwa einen Tag vorher wurden die Tiere mit Moina genügend gefüttert, und nachdem sie die Futterreste ausgespuckt hatten, für die Versuche verwandt.

Für die Herstellung der Lösungen wurde aus Glas doppelt desti- 
liertes Wasser angewandt, und vor dem Gebrauch mit $1 / 10 \mathrm{~N} \mathrm{NaHCO} 3 \mathrm{PH}$ zu 7,0 reguliert. Eine Spur von Kupfer zeigt merkliche olygodinamische Wirkung.

\section{Versuchsergebnisse.}

Um die günstigiste Salzkonzentration für Hydra zunächst festzustellen, wurde Herbst's künstliches Meerwasser in verschieden verdünnten Konzentrationen versucht; hat sich die 1/100 verdünnung am günstigsten bewährt. Diese Konzentration ist $\mathrm{zu}$ ca. $1 / 200 \mathrm{M} \mathrm{NaCl}$ isotonisch.

Als Kationen wurden $\mathrm{Na}^{*}, \mathrm{~K} \cdot \mathrm{Ca} \cdot, \mathrm{Mg}^{*}$ und als Anion nur $\mathrm{Cl}^{\prime}$ behandelt. Je zu $1 / 200 \mathrm{M} \mathrm{NaCl}$ isotonische reine Lösungen von $\mathrm{NaCl}$, $\mathrm{KCl}, \mathrm{MgCl}_{2}$ bzw. $\mathrm{CaCl}_{2}$ wurden untersucht, und alle zeigten sich stark giftig. Die Giftigkeit war am starksten bei $\mathrm{KCl}$. Der völlige Zerfall der Hydren wurde in der reinen Lösung von $\mathrm{KCl}$ innerhalb eines Tages herbeigeführt.

Durch die Vermischung der $\mathrm{zu} 1 / 200 \mathrm{M} \mathrm{NaCl}$ isotonischen reinen Lösungen von $\mathrm{NaCl}, \mathrm{KCl}, \mathrm{MgCl}_{2}$ und $\mathrm{CaCl}_{2}$, konnte ich die Lösungen, die $\mathrm{zu} 1 / 200 \mathrm{M} \mathrm{NaCl}$ immer isotonisch aber in verschiedenem Verhältnis von Ionenkonzentration, herstellen.

Bei der Vergleichung der Beeinflussung dieser Lösungen, entstand Schwierigkeit bezüglich der Identifizierung der Stufes des Depressionsprozesses jedes Exemplares mit dem der genannten Figuren, da der Depressionsprozess nicht immer bei allen Individuen von derselben Flasche noch in allen Tentakeln von demselben Individuum gleichzeitig fortschritt.

Tatsächlich wurde der Ausschlag in folgender Weise gegeben; wenn die Merkmale der nächsten Stufe der Depression in einem Exemplare auf mehr als drei Tentakeln auftrat, reihte ich es in die nächste Stufe ein, und die Giftigkeit der verschiedenen Lösungen wurde mit der Zahl $\mathrm{M}$ in der folgenden Formel, am siebten Tag des Experimentes, relativ dargestellt.

$$
\mathrm{M}=\sum \mathrm{S} \times \mathrm{N}
$$

$\mathrm{S}=$ Nummer der Stufe der Depression

$\mathrm{N}=$ Anzahl von Individuen in jeder Stufe der Depression

In solcher Weise wurden zunächst folgende Lösungen untersucht: I) Wasserleitungswasser von der Fakultät für Naturwissenschaften, Hokkaido Kaiserliche Universität (I in der Tabelle); II) Das Gemisch von den genannten 4 Stammlösungen (zu $1 / 200 \mathrm{M} \mathrm{NaCl}$ isotonische reine Lösungen von $\mathrm{NaCl}, \mathrm{KCl}, \mathrm{CaCl}_{2}$ bzw. $\mathrm{MgCl}_{2}$ ) in dem Verhältnis, dass die 4 Kationen in dem Verhältnis zueinander stehen, das dem des Seewasser entspricht (II in der Tabelle); III) Das ternäre Gemisch von den Stammlösungen $\left(\mathrm{NaCl}, \mathrm{KCl}\right.$ und $\left.\mathrm{CaCl}_{2}\right)$ in dem Verhältnis, das für die Wundheilung u. s. w. von Planarien am besten geeignet ist (nach Murray) (III in der Tabelle); IV) Das Gemisch von 4 Stammlösungen in dem Verhältnis, dass das Verhältnis der 4 Kationen dem der Lösunge I entspricht (nach der Analyse von Y. Kusuki) (IV in 
der Tabelle); V) Das ternäre Gemisch der Stammlösungen in dem für die Planarien schädlichen $\mathrm{Ca} *$ und $\mathrm{K}^{*}$ verhältnis (nach Murray) (V in der Tabelle).

TABeLle 1.

\begin{tabular}{|c|c|c|c|c|c|c|c|c|c|}
\hline \multirow[t]{2}{*}{$\begin{array}{l}\text { NR. DER } \\
\text { LÖSUN- } \\
\text { GEN }\end{array}$} & \multicolumn{4}{|c|}{$\begin{array}{l}\text { KONZENTRATIONSVERHÄLTNIS } \\
\text { JEDER KATIONEN, K' } \\
\text { ALS } 1 \text { GESETZT }\end{array}$} & \multicolumn{4}{|c|}{$\begin{array}{c}\text { PROZENTSATZ DER KONZENTRA- } \\
\text { TION JEDER KATIONEN ZUR } \\
\text { GESAMMTKONZENTRATION } \\
\text { DER KATIONEN }\end{array}$} & \multirow[t]{2}{*}{$\begin{array}{c}\text { ERGEB- } \\
\text { NISSE }\end{array}$} \\
\hline & $\mathrm{Na}^{\circ}$ & $\mathrm{Ca} *$ & $\mathrm{~K}^{\cdot}$ & Mg•• & $\mathrm{Na}^{\circ}$ & $\mathrm{Ca} *$ & $\mathrm{~K}^{\cdot}$ & Mg*• & \\
\hline I & \multicolumn{8}{|c|}{ Wasserleitungswasser von Fakul., Naturwiss., Hokkaido Kais. Univ. } & ++ \\
\hline II & 46 & 1 & 1 & 5 & 87 & 2 & 2 & 9 & ++ \\
\hline III & 90 & 3 & 1 & 0 & 96 & 3 & 1 & 0 & - \\
\hline IV & 24 & 13 & 1 & 4 & 57 & 31 & 2 & 10 & ++ \\
\hline V & 18 & 0.05 & 1 & 0 & 94 & $!$ & 6 & 0 & --- \\
\hline VI & 25 & 50 & 1 & 5 & 31 & 62 & 1 & 6 & + \\
\hline VII & 25 & 10 & 1 & 5 & 61 & 25 & 2 & 12 & ++ \\
\hline VIII & 25 & 1 & 1 & 5 & 78 & 3 & 3 & 16 & +++ \\
\hline IX & 25 & 0.1 & 1 & 5 & 80.5 & 0.3 & 3 & 16.2 & -- \\
\hline $\mathrm{X}$ & 25 & 0 & 1 & 5 & 81 & 0 & 3 & 16 & -- \\
\hline $\mathrm{XI}$ & 75 & 0.01 & 1 & 5 & 93 & $!$ & 1 & 6 & - \\
\hline XII & 40 & 0.01 & 1 & 5 & 87 & $!$ & 2 & 11 & -- \\
\hline
\end{tabular}

Die Ergebnisse sind in der Tabelle zusammengestellt (von I zu V). Es ist ganz interessant, dass, wie wir in der Tabelle bei III sehen, die für Planarien am besten geeignete Lösung für Hydren schädlich wirkt. Die stark giftige Beeinflussung der Lösung V gibt einen Hinweis, dass es antagonistische Wirkung der Ionen, einwertiger gegenüber zweiwertiger, gibt. Das dem Seewasser bzw. Leitungswasser entsprechende Salzgemisch erweist sich ziemlich passend, das Leben der Hydren zu erhalten.

In der Absicht best geeignetes Medium zu gewinnen, wurde die Versuche, unter dem Leitungswasser entsprechendem Verhältnis von $\mathrm{Na}{ }^{*}$ und $\mathrm{Mg}^{*}$ gegenüber $\mathrm{K}^{*}$ das optimale Verhältnis von $\mathrm{Ca} \cdot$ festzustellen, denn bei dem Versuche der reinen Lösungen war es nachgewiesen, dass die Giftigkeit von $\mathrm{KCl}$ besonders stark ist, und ausserdem da von M. R. Murray (1928) bei Planarien und von E. Berger (1929) bei Daphnien und anderen antagonistische, also entgiftende Wirkung von $\mathrm{Ca}^{*}$ gegenüber $\mathrm{K}^{*}$ beobachtet ist. Die Ergebnisse davon sind in der Tabelle von VI zu X dargestellt.

Der merkwürdige Antagonismus zwischen $\mathrm{Ca}{ }^{*}$ und $\mathrm{K} \cdot$ wurde auch bei Hydra festgestellt. Unter dem beständigen Verhältnis von $\mathrm{Na}^{*}$ und $\mathrm{Mg}^{*}$ gegenüber $\mathrm{K}^{*}$ wurde bei dem bestimmten Verhältnis von $\mathrm{Ca}^{*}$ 
die optimalen Bedingungen für die Lebenstätigkeit der Hydren dargestellt, und bei dem höheren bzw. niedrigeren Verhältnis von Ca* als das optimale Verhältnis trat die schädliche Wirkung auf. Die Schädlichkeit war besonders heftig bei niedrigeren Verhältnissen von $\mathrm{Ca}$ *. Unter dem der Lösung IV entsprechende $\mathrm{Na}^{*}$ und $\mathrm{Mg}^{*}$ gegenüber $\mathrm{K}^{\bullet}$ konnte ich bei dem optimalen Verhältnis von $\mathrm{Ca} *$ (VIII in der Tabelle) zur günstigsten Lebensbedingung für Hydra gelangen; Hydren haben dabei keine Neigung zur Depression gezeigt, wenigstens in den sieben Tage der Experimentdauer. Es zeigt sich als besser geeignet als die dem Seewasser entsprechende Ionenzusammensetzung.

Solches am besten für Hydra geeignetes Kulturmedium wird durch die Mischung von den genannten Stammlösungen in dem Volumenverhältnis, $25 \mathrm{NaCL}: 1,5 \mathrm{CaCl}_{2}: 1 \mathrm{KCl}: 7,5 \mathrm{MgCl}_{2}$, hergestellt, und dabei stehen die Konzentrationen der 4 Kationen zueinander im Verhältnis $25 \mathrm{Na}^{\bullet}: 1 \mathrm{~K}^{\bullet}: 1 \mathrm{Ca}^{*}: 5 \mathrm{Mg}^{*}$.

Die Lösungen XI und XII wurden so hergestellt, dass die absolute Konzentration von $\mathrm{K}^{\cdot}$ je zur Lösung IV und VI gleich ist. Darin bestätigt es sich, dass bei der dem optimalen Medium gleichen absoluten Konzentration von $\mathrm{K}^{*}$ bei relativ niedrigerer Konzentration von $\mathrm{Ca}^{*}$ die Schädlichkeit auftrit.

\section{Zusammenfassung.}

1) Als das Kontrollmedium für die Experimente der Beeinflussung der Ionen im äusseren Medium auf Hydra, wurde das Medium von günstigster Zusammensetzung von Ionen für Hydra gesucht.

2) Unter den reinen Lösungen von $\mathrm{NaCl}, \mathrm{KCl}, \mathrm{CaCl}_{2}$ und $\mathrm{MgCl}_{2}$ war die Giftigkeit bei der $\mathrm{KCl}$ am stärksten.

3) Entgiftung von $\mathrm{Ca}$ * gegenüber $\mathrm{K}$ * wurde auch bei Hydra nachgewiesen.

4) Das Gemisch der zum $1 / 200 \mathrm{M}$ isotonischen Lösungen von $\mathrm{NaCl}$, $\mathrm{KCl}, \mathrm{CaCl}_{2}$ und $\mathrm{MgCl}_{2}$ in dem Verhältnis der Kationen $25 \mathrm{Na}^{\circ}: 1 \mathrm{Ca}^{*}$ : $1 \mathrm{~K}^{\bullet}: 5 \mathrm{Mg}^{*}$ ist als das günstigste Medium für die Hydrenkultur gefunden.

5) Das genannten Salzgemisch ist besser geeignet für Hydren als das dem Seewasser entsprechende Gemisch.

\section{Literatur.}

Berger, E. 1929 Unterschiedliche Wirkungen gleicher Ionen und Ionengemisch auf verschiedenen Tierarten. Pflüg. Arch. 223.

Bethe, A. 1927 Der Einfluss der Ionen des Seewasser auf rhythmische Bewegung von Meerestieren. Pflüg. Arch. 217.

Murray, M. R. 1928 The Calcium-potassium ratio in culture media for Planaria dorotocephala. Phys. Zoöl. 1.

Palmhert, H. W. 1933 Beiträge zum Problem der Osmoregulation einiger Hydroidpolypen. Zool. Jahrb. Phys. 53. 